\title{
Solvent Effect on Structural Formation and Molecular Mobility of Polyvinyl Chloride Gels
}

\author{
Po-Da Hong ${ }^{\dagger}$ and Hsing-Tsai Huang \\ Department of Textile and Polymer Engineering, National Taiwan University of \\ Science and Technology, Taipei 10607, Taiwan
}

(Received March 29, 2000; Accepted June 5, 2000)

\begin{abstract}
In this work, the interaction between polyvinyl chloride (PVC) and organic solvents was first investigated through viscometric results and the donor-acceptor electron properties. The results clearly showed that the affinity of nitrobenzene $(\mathrm{NrBz})$ to $\mathrm{PVC}$ is higher than that of chlorobenzene $(\mathrm{ClBz})$. On the other hand, the critical gel concentrations $C_{\mathrm{gel}}{ }^{*}$ are $1.7 \mathrm{~g} \mathrm{dL}^{-1}$ and $6 \mathrm{~g} \mathrm{dL}^{1}$, respectively, for the $\mathrm{PVC} / \mathrm{ClBz}$ and $\mathrm{PVC} / \mathrm{NrBz}$ solutions at $30^{\circ} \mathrm{C}$, indicating that the polymer-solvent interaction directly affects the gelation ability. Then, the structural formation and the molecular mobility of PVC gels were investigated using pulsed ${ }^{1} \mathrm{H}$ NMR analyses. The CPMG decaying signals from pulsed NMR measurement of the PVC gels could be decomposed into three components, reflecting respectively the proton mobility in the junction zone, in the polymer-rich phase and in the solvent-rich phase of the gel network. The solutions began to appear the fraction of junction zones at PVC concentration above $1.5 \mathrm{~g} \mathrm{dL}^{-1}$ and $6 \mathrm{~g} \mathrm{dL}^{-1}$, respectively, in the $\mathrm{PVC} / \mathrm{ClBz}$ and $\mathrm{PVC} / \mathrm{NrBz}$ solutions, while the formation of the junction zones could interconnect the polymer chains into a three-dimensional network structure macro-domain. As the immobile junction zones were formed, the mobility of the PVC chains was reduced and the mobility of the solvent molecules could also be suppressed. This phenomenon typically appeared in the polymer/poor solvent (PVC/ClBz) system. Through the observation of the diffusion property of the solvent molecules, we can clearly show that the gel network in $\mathrm{PVC} / \mathrm{ClBz}$ gels is denser than that of $\mathrm{PVC} / \mathrm{NrBz}$ gels.
\end{abstract}

KEY WORDS Polyvinyl Chloride/Physical Gel/Chain Mobility/Pulsed Nuclear Magnetic Resonance /

It is well known that polyvinyl chloride (PVC) is a polar and crystalline polymer, which can form physical polymer gels in a variety of solvents possessing fringed micelle microcrystals as junction points in the network structure. ${ }^{1-10}$ During the gelation process, the polymer solution first undergoes liquid-liquid phase separation to be decomposed into two phases, a polymer-rich phase and a solvent-rich phase, then produces a number of junction points from the associations of the PVC syndiotactic sequences in the polymer-rich phase. Herein, the degree of the polymer-solvent interaction is an important factor on the gelation ability and the structural formation of the gel. The rate and degree of the liquid-liquid phase separation, which mainly controls the gelation behavior and the structure of the gel network, much depends on the solvent used. Therefore, it is well recognized that the chain mobility for polymer or solvent in solution or gel state is deeply related to the structural construction. Generally, in a polymer/poor solvent system a significant degree of the phase separation and the association of the polymer chains should easily proceed to promote gelation.

Recently, the heterogeneous structures of multicomponent polymer systems (e.g., polymer gels, polymer blends, and polymer solutions) have been studied by pulsed NMR. ${ }^{11-13}$ The decaying signals of heterogeneous materials in pulsed NMR measurement could be decomposed into two or three components having different spin-lattice $\left(T_{1}\right)$ or spin-spin $\left(T_{2}\right)$ relaxation times, indicating that the difference in chain mobility of each component. Guenet et $a l .{ }^{14}$ discussed the physical association of the syndiotactic poly(methyl methacrylate) (s-PMMA) gels by high-resolution ${ }^{1} \mathrm{H}$ NMR spectra. Their results showed a significant reduction of the absolute integrated intensities of the s-PMMA sequences during gelation. Hayashi et $a l .{ }^{15,16}$ also found the reductions in the mobility of polymer chains and solvent molecules during the volume phase transition of the acrylamide gels. Watanabe $e t a l .{ }^{17}$ also discussed the multi-exponential proton relaxation process of compartmentalized water in Sephadex gels through pulsed NMR analysis. The Sephadex gels revealed the existence of two relaxation components indicating two non-exchangeable water components, i.e., water in the pores and water associated with the junction zones in the gel network. As stated earlier, the polymer-solvent interaction must predominantly control the structural formation of the gel network. Therefore, both the mobility of polymer chains and solvent molecules should be changed significantly when the polymer solution undergoes phase separation or gelation.

In this work, regarding the molecular structures of each component, the polar hydrogen and polar chlorine atoms on the $\mathrm{H}^{\delta+}-\mathrm{C}-\mathrm{Cl}^{\delta-}$ part of PVC could act as electron acceptors and electron donors, respectively. The molecular structures of the used solvents, chlorobenzene $(\mathrm{ClBz})$ and nitrobenzene $(\mathrm{NrBz})$, with various functional groups could represent different phenomena on the delocalizing of the intramolecular charge density. The heteronuclear substrates $\mathrm{A}^{\delta+}-\mathrm{B}^{\delta-}$ of the solvents could be attacked by the electron donors (i.e., the $\mathrm{Cl}^{\delta}$-atoms of PVC) and the electron acceptors (i.e., the $\mathrm{H}^{\delta+}$ atoms of PVC) with the low electron density regions, $\mathrm{A}^{\delta+}$ and high electron density regions, $\mathrm{B}^{\delta-}$, respectively. Various kinds of solvent used should induce different donor-acceptor properties between polymer and solvent, resulting in different degrees of polymer-solvent interaction. In this study, the affinity properties between PVC and the used solvents are first discussed. Then, the effect of

\footnotetext{
${ }^{\dagger}$ To whom correspondence should be addressed (Tel: +886-2-27376539, Fax: +886-2-27376544, E-mail: phong@hp730.tx.ntust.edu.tw).
} 
the polymer-solvent interaction on the gelation behavior and the gel structure are investigated. The variation in the mobility of PVC chains and solvent molecules during the gelation process as disclosed by pulsed ${ }^{1} \mathrm{H}$ NMR analyses are also discussed.

\section{EXPERIMENTAL}

\section{Sample Preparation}

The PVC used in this work was laboratory grade powder with weight-average molecular weigh of $50.0 \times 10^{4}$ $\mathrm{g} \mathrm{mol}^{-1}$ (Aldrich Chemical Co. Ltd., USA). The ${ }^{13} \mathrm{C}$ NMR determination of tacticity for PVC has given the following result for the triads: iso $=0.20$, syndio $=0.32$ and hetero $=0.48$. The solvents, chlorobenzene $(\mathrm{ClBz})$ and nitrobenzene $(\mathrm{NrBz})$, were analytical grade and purified by distillation before using. The PVC solutions were prepared in a wide mouth bottle, with stirring, below the boiling point of the solvent, for two hours until dissolved into homogeneous solutions. Then, the solutions were cooled to $30^{\circ} \mathrm{C}$ for one day for gelation.

\section{Measurements}

The viscosities of dilute PVC solutions were determined using an Ubbelohde viscometer immersed into a thermostatic water bath held at $30^{\circ} \mathrm{C}$. Through the classical Huggins equation, ${ }^{18}$ the intrinsic viscosity $[\eta]$ and the Huggins constant $k$ could be obtained, where $t$ and $t_{0}$ are, respectively, the flowing times of the polymer solution and the pure solvent; $\eta_{\mathrm{sp}}$ is the specific viscosity.

$$
\frac{\left(t-t_{0}\right) / t_{0}}{C}=\left(\eta_{\mathrm{sp}} / C\right)=[\eta]+k[\eta]^{2} C
$$

The gelation time, $t_{\text {gel }}$, was determined by a gelation timer (Techne Co., U. K.) operating at a fixed temperature $30^{\circ} \mathrm{C}$ with a flat weighted disc connected by a link with a synchronous motor falling under gravity in the polymer solutions. At a gelation point, the rigidity of the polymer solution is sufficient to support the weight of the disc and causes the link to be compressed and to stop the synchronous motor. The time at which the synchronous motor stopped was defined as gelation time.

The gelation temperature, $T_{\text {gel }}$, was determined by a test tube tilting method. The solution was quenched in a water-bath kept at a given temperature for one day, then test tubes were tilted. If the level meniscus deformed under its own weight, we considered that the solution was still a sol. When the meniscus could not be seen to deform, we judged that the solution had gelled.

The "test tube upside-down" method was used for determining the gel melting temperatures $T_{\mathrm{m}} \mathrm{G}$, where the gel was put in sealed glass tubes. The test tube with gel was kept upside down in a thermostat oven with a heating rate of $1^{\circ} \mathrm{C} \min ^{-1}$ to keep the gel in the test tube heated evenly. The temperature at which the gel began to flow was defined as $T_{\mathrm{m}} \mathrm{G}$.

The pulsed ${ }^{1} \mathrm{H}$ NMR measurements were performed by a MARAN-20 pulsed NMR spectrometer operating at a fixed frequency of $20 \mathrm{MHz}$. The recovery time of the spectrometer following a pulse sequence was $10 \mu \mathrm{s}$. The spin-spin relaxation time $\left(T_{2}\right)$ was determined by the Carr-Purcell-Meiboom-Gill (CPMG) method ${ }^{19}\left[90^{\circ}{ }_{x} \tau\right.$ $\left.\left(180^{\circ}{ }_{y} 2 \tau\right)_{n}\right]\left(\tau=50 \mu \mathrm{s}, \mathrm{P} 90^{\circ}=2.9 \mu \mathrm{s}, n=4096\right)$ for eli-

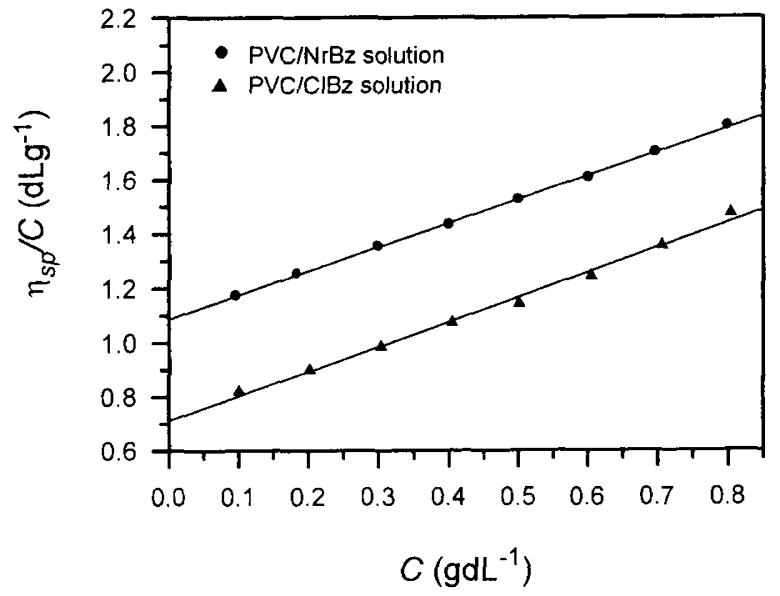

Figure 1. Plot of the specific viscosity $\eta_{\mathrm{sp}}$ versus PVC concentration $C$ at $30^{\circ} \mathrm{C}$.

minating the effect of the heterogeneity in the static magnetic field. The CPMG pulsed sequence was available for the long $T_{2}$ samples, e.g., the wet gels.

\section{Analysis of NMR Decaying Signals}

The Weibull function could be used to express the decaying of the transverse magnetization intensity, $M(t)^{20,21}$

$$
M(t)=M_{0} \exp \left[-(1 / a)\left(t / T_{2}\right)^{a}\right]
$$

Where, $M_{0}$ is a constant proportional to the total number of nuclei with magnetic moment, $T_{2}$ is the spin-spin relaxation time and $t$ is the decay time. The $a$ factors are the shape parameters, which could be divided into a hard (immobile) component and a soft (mobile) component for $a=2$ (Gaussian decay) and $a=1$ (exponential decay), respectively. As the heterogeneous gel led to heterogeneity of the molecular motion, the CPMG signals could be decomposed into several decaying components with various $T_{2}$ values, as given in eq 3 .

$$
M(t)=\sum M_{0}^{i} \exp \left[-(1 / a)\left(t / T_{2}^{i}\right)^{a}\right]
$$

The $M_{0}{ }^{i}$ is the magnetic moment fraction of the $i$ th component and the fractional amount of each component, $F^{i}$, is equal to $M_{0}{ }^{i} / \Sigma M_{0}{ }^{i}$ value.

\section{RESULTS AND DISCUSSION}

Figure 1 shows the plots of the reduced viscosity $\eta_{\mathrm{sp}} / C$ as a function of the PVC concentration $C$ for the PVC/ $\mathrm{NrBz}$ and $\mathrm{PVC} / \mathrm{ClBz}$ solutions at $30^{\circ} \mathrm{C}$. Then, the Huggins constants $k$ and the intrinsic viscosities $[\eta]$ could be obtained through the calculation of eq 1 . The values of $k$ and $[\eta]$ are $0.74,1.09 \mathrm{dL} \mathrm{g}^{-1}$ and $1.80,0.71 \mathrm{dL} \mathrm{g}^{-1}$ for $\mathrm{PVC} / \mathrm{NrBz}$ and $\mathrm{PVC} / \mathrm{ClBz}$ solutions, respectively. Generally, the Huggins constant ( $k$ value) could be used to predict the degree of the polymer-solvent interaction in a binary polymer solution. In a good solvent system, $k<$ 0.52 , the polymer chains exhibit relatively extended conformations. In a $\theta$ solvent system, $k=0.52$, the polymer chains are unperturbed coils. In a poor solvent system, $k>0.8$, the polymer chains were collapsed and intra- 
molecular aggregation occurred easily. In this study, the $k$ values for the $\mathrm{PVC} / \mathrm{NrBz}$ and $\mathrm{PVC} / \mathrm{ClBz}$ solutions are 0.74 and 1.80 , respectively, indicating that the affinity of the $\mathrm{NrBz}$ to $\mathrm{PVC}$ is higher than that of the ClBz. It is well known that the molecular interaction between a polar polymer and a polar solvent cannot be estimated directly by the solubility parameters $\delta$. Two polar solvents used in this study have similar molar volumes $V$ and solubility parameters $\delta, \mathrm{ClBz}\left(v=107 \mathrm{~cm}^{3} \mathrm{~mol}^{-1} ; \delta=\right.$ $\left.9.5 \mathrm{cal}^{0.5} \mathrm{~cm}^{-1.5}\right)$ and $\mathrm{NrBz}\left(v=103 \mathrm{~cm}^{3} \mathrm{~mol}^{-1} ; \delta=10\right.$ $\mathrm{cal}^{0.5} \mathrm{~cm}^{-1.5}$ ), and PVC has the solubility parameter $\delta=$ $9.6 \mathrm{cal}^{0.5} \mathrm{~cm}^{-1.5}$. Obviously, these solubility parameters cannot be used to clarify the interaction between PVC and solvent. Therefore, we directly focus on the molecular structure and the donor-acceptor electron property between each component for understanding the molecular interaction.

The $\mathrm{H}^{\delta+}-\mathrm{C}_{-} \mathrm{Cl}^{\delta-}$ parts of the PVC chains with the polar $\mathrm{H}^{\delta+}$ atoms and the polar $\mathrm{Cl}^{\delta-}$ atoms could act respectively as the electron acceptors and donors, which could be strongly attacked by the heteronuclear substrates, $\mathrm{A}^{\delta+}-\mathrm{B}^{\delta-}$, of the solvent molecules. The $\mathrm{Cl}^{\delta-}$ atoms on the PVC chains could be attacked by the lower electron density regions of the solvent molecules, $\mathrm{A}^{\delta+}$, on the other hand, the $\mathrm{H}^{\delta+}$ atoms of PVC could be attacked by higher electron density regions, $\mathrm{B}^{\delta-}$. Particularly, the functional groups in the substituted benzene mainly dominate the heteronuclear $\mathrm{A}^{\delta+}-\mathrm{B}^{\delta-}$ properties of solvents. In the $\mathrm{NrBz}$ molecule, the nitro group, $\mathrm{NO}_{2}$, tends to withdraw electrons inductively. This inductive effect (I-effect) could delocalize the electrons from the benzene ring to the oxygen atom. Additionally, the dipolar resonance structures in $\mathrm{NrBz}$ also place positive charges on the benzene ring. ${ }^{22}$ Both effects could be present in the following schemes. Therefore, the $\mathrm{NrBz}$ molecules tend to an explicit heteronuclear $\mathrm{A}^{\delta+}-\mathrm{B}^{\delta-}$ property to induce higher donor-acceptor electron activity between PVC and $\mathrm{NrBz}$.

\section{I-effect Resonance effect}

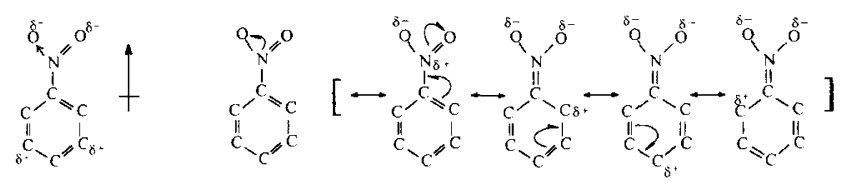

In the $\mathrm{ClBz}$ molecule, the hyperconjugation effect (Meffect), indicates the overlapping between the $2 p$ orbital on chlorine and the $\pi$ orbital on benzene, while resigning the electrons from the chlorine atom to the benzene ring. ${ }^{22}$ This M-effect could reduce the contribution of the inductive effect (I-effect) to induce this halogen benzene possessing unapparent heteronuclear property, reflecting a lower donor-acceptor electron property between PVC and ClBz.

\section{I-effect}<smiles>Clc1ccccc1</smiles>

\section{M-effect}

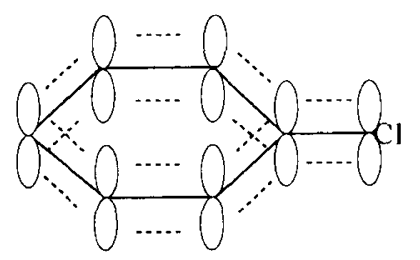

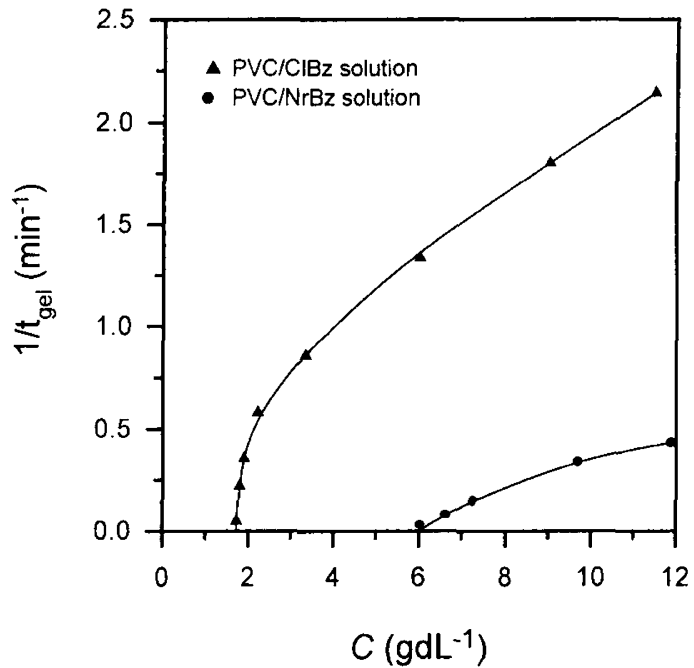

Figure 2. The gelation rate $1 / t_{\mathrm{gel}}$ as a function of $C$ at $30^{\circ} \mathrm{C}$.

Figure 2 represents the gelation rate, $1 / t_{\text {gel }}$, as a function of $C$, where $t_{\text {gel }}$ is the gelation time. At a given concentration, the gelation rate of the $\mathrm{PVC} / \mathrm{ClBz}$ solution is faster than that of the PVC/NrBz one. During the gelation process, the polymer solution should first decompose into two phases, a polymer-rich phase and a solvent-rich phase, then the PVC syndiotactic sequences of the polymer-rich phase associate with each other to form junction points. Eventually, these junction points interconnect the PVC chains into an infinite threedimensional network structure macro-domain. This gelation mechanism of the PVC solutions has been verified by laser light-scattering experiments and the spinodal decomposition theory. ${ }^{23}$ In a polymer/poor solvent $(\mathrm{ClBz})$ system, the liquid-liquid phase separation and PVC chains aggregation should proceed easily, while resulting in a rapid gelation rate. Moreover, the gelation of the PVC solutions could occur at the concentration above a critical gel concentration, $C_{\text {gel }}^{*}$, obtained from the extrapolation of the zero gelation rate, $1 / t_{\text {gel }}=0$ in Figure 2. The values of $C_{\text {gel }}^{*}$ are $c a .1 .7 \mathrm{~g} \mathrm{dL}^{-1}$ and $6.0 \mathrm{~g} \mathrm{dL}^{-1}$ respectively for the $\mathrm{PVC} / \mathrm{ClBz}$ and $\mathrm{PVC} / \mathrm{NrBz}$ solutions, directly indicating that the gelation of $\mathrm{PVC} / \mathrm{NrBz}$ solution is relatively difficult because of the higher affinity of $\mathrm{NrBz}$ to PVC.

Figure 3 shows the gel melting temperature $\left(T_{m}{ }^{G}\right)$ and the sol-gel transition temperature $\left(T_{\text {gel }}\right)$ as a function of $C$ in the $\mathrm{PVC} / \mathrm{ClBz}$ and $\mathrm{PVC} / \mathrm{NrBz}$ solutions. Both the $T_{\mathrm{m}}{ }^{\mathrm{G}}$ and $T_{\text {gel }}$ values rise with $C$, indicating directly that the increasing concentration is advantageous to gelation. At a given concentration, the $\mathrm{PVC} / \mathrm{ClBz}$ gel has higher $T_{\mathrm{m}}{ }^{\mathrm{G}}$ and $T_{\text {gel }}$ values compared to $\mathrm{PVC} / \mathrm{NrBz}$ gel. The $T_{\text {gel }}$ results imply the total free energy for the breaking of the PVC-solvent associations and the association of the PVC sequences in the PVC/ClBz solutions could be a thermodynamic stability at higher temperature. The $T_{\mathrm{m}}{ }^{\mathrm{G}}$ results could be explained clearly by a Flory-Huggins formalism ${ }^{24}$ for the melting point depression, the gel has a lower $T_{\mathrm{m}}{ }^{\mathrm{G}}$ value for higher polymer-solvent affinity. Particularly, this phase diagram presents a hysteresis process at the temperature range from $T_{\text {gel }}$ to $T_{\mathrm{m}}{ }^{\mathrm{G}}$. In other systems, similar hysteresis phenomena can also be found, such as the atactic polystyrene/CS 


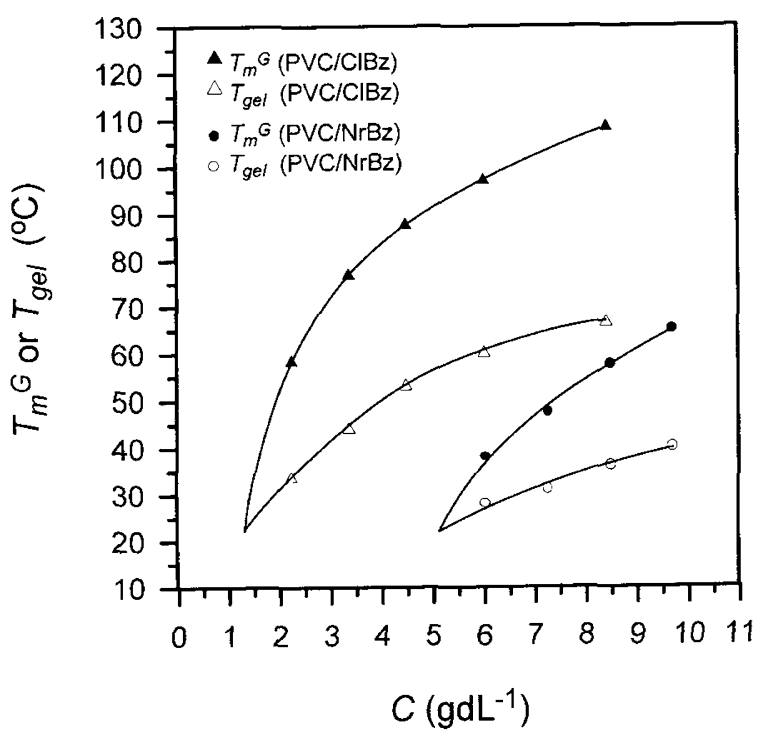

Figure 3. The gel melting temperature $T_{\mathrm{m}}{ }^{\mathrm{G}}$ and the sol-gel transition temperature $T_{\mathrm{gel}}$ as a function of $C$.

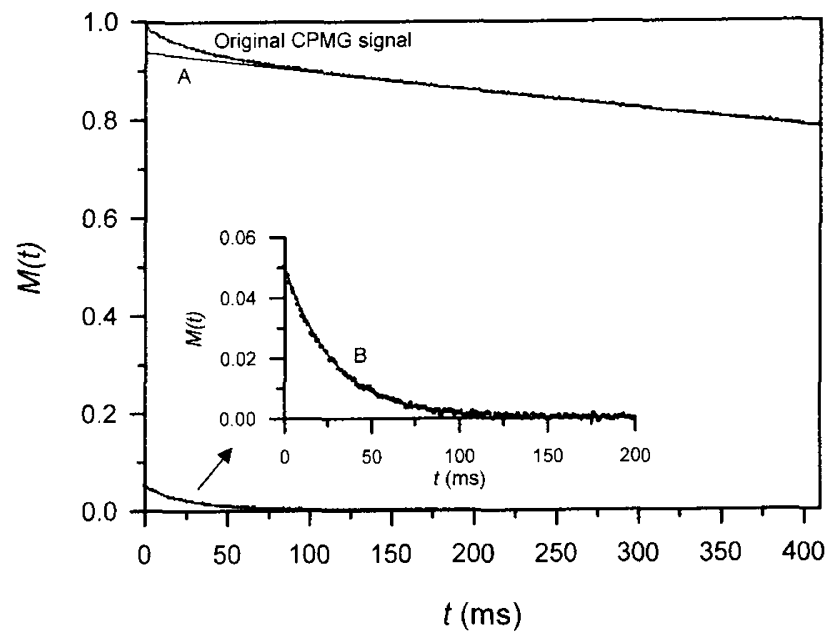

Figure 4. The CPMG decaying signal of PVC/NrBz $\left(5 \mathrm{~g} \mathrm{dL}^{-1}\right)$ system at $30^{\circ} \mathrm{C}$.

(at-PS/ $\mathrm{CS}_{2}$ ) system. ${ }^{25}$ The combination of the $T_{\mathrm{m}}{ }^{\mathrm{G}}$ and $T_{\text {gel }}$ curves could directly divide the phase diagram into three regions, the solution region is the state above the $T_{\mathrm{m}}{ }^{\mathrm{G}}$ curve; the sol region is the state between the $T_{\mathrm{m}}{ }^{\mathrm{G}}$ and $T_{\text {gel }}$ curves; the gel region is the state below the $T_{\text {gel }}$ curve. At a given concentration, a relatively long temperature range in the $\mathrm{PVC} / \mathrm{ClBz}$ system for sol-gel transition indicates this sample is more heterogeneous. The solution shows no remarkable heterogeneity in a particular concentration where $T_{\mathrm{m}}{ }^{G}$ is equal to $T_{\mathrm{gel}}$. These estimated concentrations are $1.3 \mathrm{~g} \mathrm{dL}^{-1}$ and $5 \mathrm{~g} \mathrm{dL}^{-1}$ for $\mathrm{PVC} / \mathrm{ClBz}$ and $\mathrm{PVC} / \mathrm{NrBz}$ solutions, respectively. This result illustrates that the concentration-dependence of the heterogeneity of $\mathrm{PVC} / \mathrm{ClBz}$ samples is more significant than that of $\mathrm{PVC} / \mathrm{NrBz}$ ones.

As mentioned above, the PVC-solvent interactions seem to significantly correlate with the gelation capability and the heterogeneity of the gels. In the following study, the heterogeneity of the PVC samples was investigated through pulsed NMR analyses. Figure 4 shows a

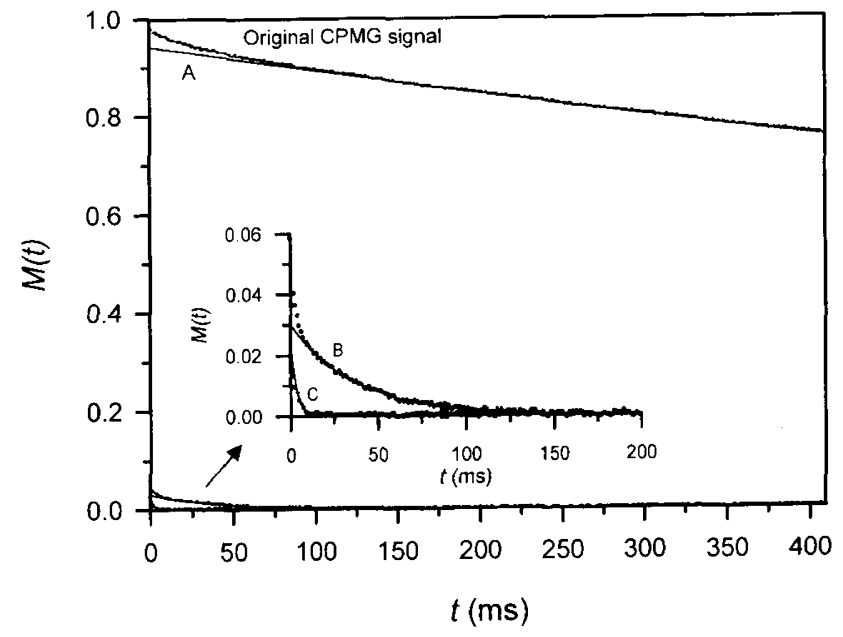

Figure 5. The CPMG decaying signal of the PVC/ClBz $\left(5 \mathrm{~g} \mathrm{dL}^{-1}\right)$ system at $30^{\circ} \mathrm{C}$

CPMG decaying signal for $\mathrm{PVC} / \mathrm{NrBz}$ sample with the concentration of $5 \mathrm{~g} \mathrm{dL}^{-1}$ at $30^{\circ} \mathrm{C}$. This decaying signal can be decomposed into two exponential decaying components, while the components $A$ and $B$ are the protons with various spin-spin relaxation time $\left(T_{2}\right)$ in the solvent-rich and in the polymer-rich phases. The values of $T_{2}{ }^{A}$ and $T_{2}{ }^{B}$ are $2271 \mathrm{~ms}$ and $31 \mathrm{~ms}$, respectively. On the other hand, Figure 5 shows the CPMG signal for the $5 \mathrm{~g} \mathrm{dL}^{-1} \mathrm{PVC} / \mathrm{ClBz}$ gel at $30^{\circ} \mathrm{C}$. The third Gaussian decaying component $C$ with a very short $T_{2}$ value $\left(c a . T_{2}{ }^{\mathrm{C}}=\right.$ $1.8 \mathrm{~ms}$ ) can be separated from this decaying signal. The solutions begin to exhibit this hard component at $C>1.5$ $\mathrm{g} \mathrm{dL}^{-1}$ and $C>6 \mathrm{~g} \mathrm{dL}^{-1}$, respectively, in the $\mathrm{PVC} / \mathrm{ClBz}$ and PVC/NrBz solutions. As is well known, molecular motion with a time scale of $T_{2} \sim 0.01 \mathrm{~ms}$ reflects a polymer crystal domain which has strong dipole interaction between the spins. ${ }^{26}$ Actually, we cannot find any $T_{2}$ $0.01 \mathrm{~ms}$ component in the PVC gels even from free induction decay (FID) analysis, ${ }^{27}$ i.e., the measurement is carried out using the solid echo pulsed sequence available for a short $T_{2}$ sample, for example, the crystalline domains in gel systems. Furthermore, in our previous study ${ }^{9,28}$ the wide angle X-ray diffraction (WAXD) curves for PVC gels only present an amorphous scattering peak. Then, the WAXD intensity curves of freeze-dried PVC gels which have no remarkable shrinkage (less than $1 \%$ ) show some crystalline peaks, but the crystallinity was still very low. ${ }^{28}$ These results indicate that the microcrystals in the gel network should be quite small. Therefore, we could suppose the following process in the formation of PVC gels. First, the PVC solutions undergo the liquid-liquid phase separation to form the polymerrich and solvent-rich phases, then the syndiotactic PVC sequences in the polymer-rich phase associate with each other to produce microcrystals. Subsequently, these microcrystals and some denser chain aggregates may construct a junction zone. Then, these junction zones interconnect the PVC chains to form a three-dimensional network structure macro-domain. From the above consideration and the NMR results, the hard component $\mathrm{C}$ may be related to the molecular mobility of the protons in the junction zones, because the time scale of $T_{2}$ ( $c a .2$ $\mathrm{ms}$ ) is much longer than that of the crystalline domain 


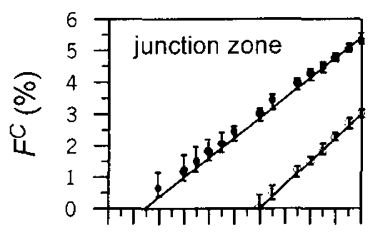

0122345678910

$C\left(\mathrm{gdL}^{-1}\right)$

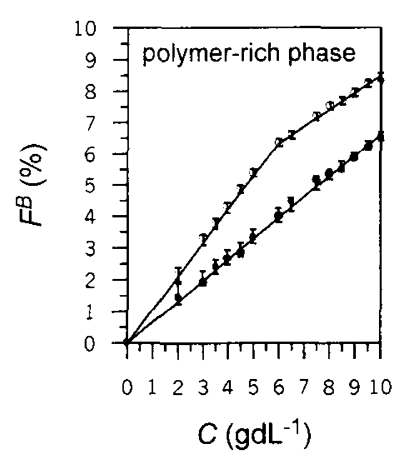

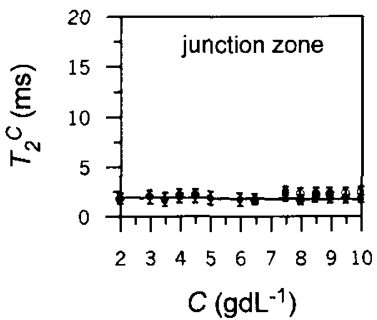

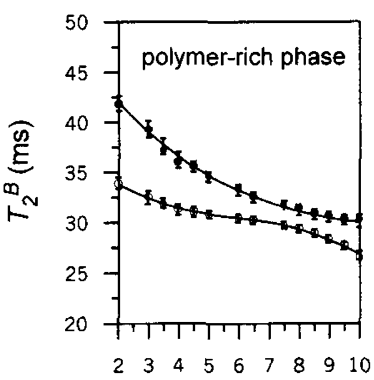

$C\left(g^{2} L^{-1}\right)$

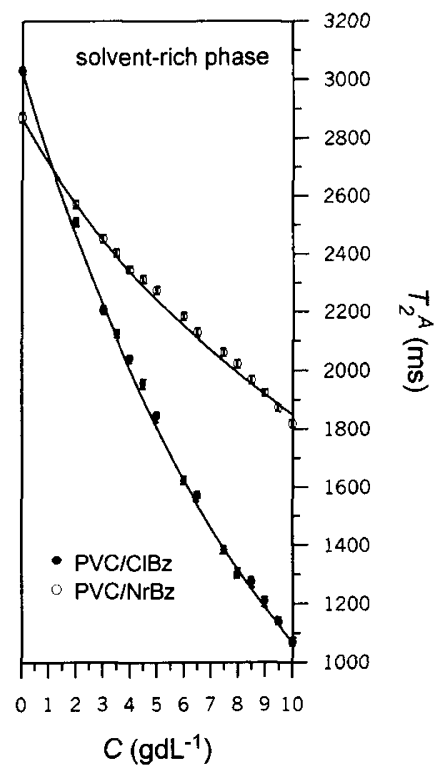

Figure 7. The spin-spin relaxation time $T_{2}$ of each component as a function of $C$.
Figure 6. The fractional amount $F$ of each component as a function of $C$.

(ca. $0.01 \mathrm{~ms}$ ) but much shorter than that of the polymerrich phase (ca. $30 \mathrm{~ms})$.

According to the above clarification for various components separated from the pulsed NMR analysis, the concentration and solvent effect on the gel structural change can be clearly presented in the following results. Figures 6 and 7 respectively show the fractional amount $F$ and the spin-spin relaxation time $T_{2}$ of each component as a function of $C$. In the $\mathrm{PVC} / \mathrm{ClBz}$ system, the construction of a larger amount of junction zones in the polymer-rich phase directly induces the higher fraction of $F^{\mathrm{c}}$. Extremely, the component of the junction zone in this $\mathrm{PVC} / \mathrm{ClBz}$ system began to appear at $C>1.5 \mathrm{~g} \mathrm{dL}{ }^{1}$; which was only found at $C>6 \mathrm{~g} \mathrm{dL}^{-1}$ in the $\mathrm{PVC} / \mathrm{NrBz}$ system. This result is in good agreement with that of the critical gel concentration $C_{\text {gel }}^{*}$ in Figure 2. This fact indicates that in $\mathrm{NrBz}$ the microcrystals or the junction zones are difficult to be formed, due to the higher affinity of $\mathrm{NrBz}$ to PVC. Only when the increment of the contact probability of PVC sequences at higher concentrations, the junction zones may be constructed. On the other hand, the aggregation of PVC chains should proceed easily in $\mathrm{ClBz}$, the poorer affinity may cause the hydrogen bonding energy between the syndiotactic sequences of PVC chains to exceed the interaction energy between PVC and ClBz. Besides, the $T_{2}{ }^{\mathrm{C}}$ values $\left(T_{2}{ }^{\mathrm{C}} \sim\right.$ $2.2 \mathrm{~ms}$ ) are almost independent of the concentration or the solvent used, as shown in Figure 7, indicating that the molecular mobility of the protons in the junction zones is very similar, regardless of the gelation conditions, i.e., the quality of the junction zones is the same.

Figure 7 also shows the $T_{2}{ }^{B}$ value which related to the chain mobility in the polymer-rich phase of the $\mathrm{PVC} /$ $\mathrm{ClBz}$ system is longer than that in the $\mathrm{PVC} / \mathrm{NrBz}$ one at a given concentration. It could be due to PVC chains exhibiting a relatively extended conformation in $\mathrm{NrBz}$, making the chain more rigid, as compared with that in a poor solvent, $\mathrm{ClBz}$. Besides, there are also two factors governing the chain mobility of the component $\mathrm{B}$, i.e., the entanglements of polymer chains and the formation of immobile junction zones. Physically, the entanglements of PVC chains directly restrict chain mobility, typically at high concentration. Secondly, a high degree of formation of immobile junction zones could additionally restrict the mobility of PVC chains. In the $\mathrm{PVC} /$ $\mathrm{NrBz}$ system, the slight reduction of the $T_{2}{ }^{B}$ values at $C$ $<6 \mathrm{~g} \mathrm{dL}^{-1}$ is mainly due to the entanglement effect. Then, the formation of the junction zones at $C>6 \mathrm{~g} \mathrm{dL}^{-1}$ could additionally reduce the $T_{2}{ }^{B}$ value, while the gelation occurs. In the PVC/ClBz system, the effect of immobile junction zones appears at lower concentration, which mainly reflecting the pronounced $T_{2}{ }^{B}$ reduction. Figure 6 also shows the fractional amount of the component $B, F^{B}$, in the $\mathrm{PVC} / \mathrm{NrBz}$ system is noticeably larger than that in the $\mathrm{PVC} / \mathrm{ClBz}$ system. The $\mathrm{PVC} / \mathrm{NrBz}$ system shows a higher $F^{B}$ value because of the formation of less amount of junction zones in the polymer-rich phase.

Subsequently, Figure 7 represents the rapid decrease in the $T_{2}{ }^{A}$ values for the $\mathrm{PVC} / \mathrm{ClBz}$ system. Primarily, it could be due to a geometric effect to reduce the solvent mobility, arising from the requirement that the solvent molecules move around the solute. ${ }^{29}$ Furthermore, the reduction of pore size in the gel network is also a factor to suppress the mobility of solvent molecules. ${ }^{30}$ The fractional amount of the protons in the solvent-rich phase $F^{A}$ linearly decreases with increasing of the PVC concentration, as shown in Figure 6. In the PVC/ClBz system, the junction zones could be easily formed to give rise to a denser gel network with smaller pore size, which could efficiently suppress the $\mathrm{ClBz}$ mobility, resulting in rapid decrease of the $T_{2}{ }^{A}$ value. In the $\mathrm{PVC} /$ $\mathrm{NrBz}$ system, only the looser network structure could be formed at high $C$, while only partially suppressing the $\mathrm{NrBz}$ mobility.

Moreover, we expected to reveal the structures of two PVC gels directly from the various chain mobilities of solvent molecules, regarding the solvent molecules as probes to investigate the solvent diffusion in the solution 


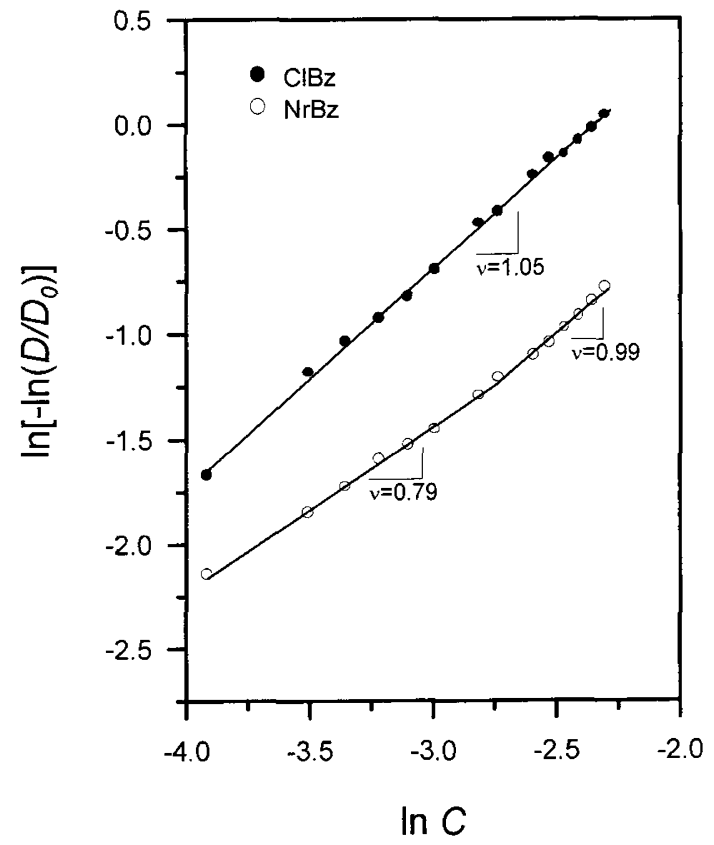

Figure 8. The plot of $\ln \left[-\ln \left(D / D_{0}\right)\right]$ versus $\ln C$.

or gel states. We must first assume that the chain mobility of the component $\mathrm{A}$ is most related to the solvent molecules, because the $F^{A}$ value in Figure 6 is quite close to the weight fraction of the solvent. The $T_{2}{ }^{A}$ of the value longer than a few seconds generally exhibits rapid mobility and short correlation time $\tau_{\mathrm{c}}\left(\tau_{\mathrm{c}}-10^{-12} \mathrm{~s}\right)$. The correlation time denotes the average time between the molecular collisions. For this rapid molecular mobility regime, the $\tau_{\mathrm{c}}$ value is much shorter than the Larmor period, $1 / \omega_{0}$, of the spins, and the inverse relaxation times were proportional to $\tau_{\mathrm{c}}{ }^{31}$

$$
\frac{1}{T_{2}{ }^{A}} \cong \frac{1}{T_{1}^{A}} \propto \tau_{\mathrm{c}}
$$

For the random motion of the solvent molecules, the diffusion coefficient $D$ could be assumed as the following equation from the derivation of the Stokes formula, ${ }^{31}$ where $l$ is the radius of the probe. It could be supposed that the $l$ values for the $\mathrm{NrBz}$ and $\mathrm{ClBz}$ molecules are quite similar, because the two solvents have similar molar volume. Then, the diffusion of solvent molecules could be directly proportional to $T_{2}{ }^{A}$ factor.

$$
D=\frac{2 l^{2}}{9} \tau_{\mathrm{c}}\left(T_{2}^{A}\right)
$$

Many studies ${ }^{32-40}$ have reported that probe diffusion depends greatly on the polymer concentration $C$, considering two parameters, $\alpha$ and $v$ as given in eq 6 , where $D$ and $D_{0}$ values denote the probe diffusion coefficients in the network media and in the neat solvent, respectively. The $\alpha$ parameter is strongly correlated with $\xi / l$ value, where $\xi$ is average pore size of the media. The rapid probe diffusion corresponds to the diffusion of solvent probes in the large $\xi$ network media, here directly representing smaller $\alpha$ value. The exponent $v$ value mainly relates to the morphology of the media and $v \approx 1$ denotes the probe diffusion in the three-dimensional network structure.

$$
\frac{D}{D_{0}}=\exp \left(-\alpha C^{v}\right)
$$

In Figure 8, the $\alpha$ and $v$ parameters are obtained from the $\log -\log$ plots of $-\ln \left(D / D_{0}\right)$ versus $C$ and yield the following stretched exponential functions.

$$
\begin{array}{ll}
\frac{D}{D_{0}}=\exp \left(-11.67 \times C^{1.05}\right) & \left(\mathrm{ClBz} \text { at } C>2 \mathrm{~g} \mathrm{dL}^{-1}\right) \\
\frac{D}{D_{0}}=\exp \left(-4.42 \times C^{0.99}\right) & \left(\mathrm{NrBz} \text { at } C>6 \mathrm{~g} \mathrm{dL}^{-1}\right) \\
\frac{D}{D_{0}}=\exp \left(-2.51 \times C^{0.79}\right) & \left(\mathrm{NrBz} \text { at } C<6 \mathrm{~g} \mathrm{dL}^{-1}\right)
\end{array}
$$

In the $\mathrm{PVC} / \mathrm{NrBz}$ system at $C<6 \mathrm{~g} \mathrm{dL}^{-1}$, the $\mathrm{NrBz}$ molecules seem to move around hindered only by the entanglement of the PVC chains for $v=0.79$. At $C<6 \mathrm{~g} \mathrm{dL}^{-1}$, the $v$ value increases to 0.99 , i.e., the media certainly changes to a network structure. In the $\mathrm{PVC} / \mathrm{ClBz}$ system, the network structure could be easily formed at lower concentration ( $c a .2 \mathrm{~g} \mathrm{dL}^{-1}$ ) where the $v$ value is persistently equal to 1.05 . The $\alpha=11.67$ in the PVC/ $\mathrm{ClBz}$ gel denotes strong retardation of the $\mathrm{ClBz}$ diffusion for this system with small $\xi / l$ value, indicating the small pore size in $\mathrm{PVC} / \mathrm{ClBz}$ gel network. On the other hand, $\alpha$ $=4.42$ in the $\mathrm{PVC} / \mathrm{NrBz}$ gels without significant reduction in $\mathrm{NrBz}$ diffusion indicate large $\xi / l$ value in this system, in other words the $\mathrm{PVC} / \mathrm{NrBz}$ network structure is looser than that in PVC/ClBz one.

\section{CONCLUSION}

In the polar polymer and polar solvent systems, we cannot discuss the degree of polymer-solvent interactions directly using the solubility parameters, but the donor-acceptor electron properties between polymer and solvent could support the results, perfectly. It could be mentioned again that the degree of the polymer-solvent interactions mainly dominated the gelation behaviors and the gel structures. From the pulsed ${ }^{1} \mathrm{H}$ NMR results, the structure of PVC gels could be decomposed into three components, component $\mathrm{A}, \mathrm{B}$, and $\mathrm{C}$ to reflect, respectively, the proton mobility in the solvent-rich phase, in the polymer-rich phase and in the immobile junction zones. The immobile junction zones in the polymer-rich phase could interconnect the PVC chains to form a three-dimensional network structure macro-domain, especially in a poor solvent system. The mobility of PVC chains could be reduced and the mobility of solvent molecules could be suppressed as immobile junction zones began to appear. Through the discussion of solvent mobility, we can clearly verify that the network structure of the $\mathrm{PVC} / \mathrm{ClBz}$ gel is denser than that of the $\mathrm{PVC} /$ $\mathrm{NrBz}$ gel, because of the poorer affinity of $\mathrm{ClBz}$ to $\mathrm{PVC}$ which makes the polymer chains aggregate easily.

Acknowledgments. The authors wish to thank the National Science Council of the Republic of China for financial aid through the project NSC-88-2216-E011-026. 


\section{REFERENCES}

1. H. Soenen and H. Berghmans, J. Polym. Sci., Polym. Phys. Ed., 34, 241 (1996).

2. H. Soenen and H. Berghmans, Polym. Gels. Netw., 2, 159 (1994).

3. Y. C. Yang and P. H. Geil, J. Macromol. Sci. Phys., B22(2), 463 (1983).

4. V. A. Kabanov and I. M. Papisov, Polym. Sci. U.S.S.R., 21, 261 (1979).

5. S. J. Candau and Y. Dormoy, Polymer, 28, 1334 (1987).

6. S. J. Guerreo and A. Keller, J. Macromol. Sci. Phys., B20(2), 161 (1981).

7. P. H. Mutin and J. M. Guenet, Macromolecules, 22, 843 (1989).

8. P. H. Mutin and J. M. Guenet, Polymer, 29, 30 (1988).

9. P. D. Hong and J. H. Chen, Polymer, 39, 711 (1998).

10. P. D. Hong and H. T. Huang, Eur. Polym. J., 35, 2155 (1999).

11. T. Shiga, K. Fukumori, Y. Hirose, A. Okada, and T. Kurauchi, J. Polym. Sci., Polym. Phys. Ed., 32, 85 (1994).

12. F. X. Quinn, E. Kampff, G. Smyth, and V. J. Mcbrierty, Macromolecules, 21, 3191 (1988).

13 H. Tanaka, K. Fukumori, and T. Nishi, J. Chem. Phys., 89, 3363(1988).

14. A. Saiani, J. Spevacek, and J. M. Guenet, Macromolecules, 31, 703 (1998).

15. T. Ikehara, T. Nishi, and T. Hayashi, Polym. J., 28, 169 (1996).

16. T. Tokuhiro, T. Amiya, A. Mamada, and T. Tanaka, Macromolecules, 24, 2936 (1991).

17. T. Watanabe, N. Murase, M. Staemmler, and K. Gersonde, Magn. Reson. Med., 27, 118 (1992).

18. M. L. Huggins, J. Am. Chem. Soc., 64, 2716 (1942).

19. S. Meiboom and D. Gill, Rev. Sci. Instrum., 29, 688, (1958).
20. S. Kaufman, W. P. Slichter, and D. D. Davis, J. Polym. Sci., A-2, 9, 829 (1971).

21. H. Tanaka and T. Nishi, J. Chem. Phys., 85, 6197 (1986).

22. K. P. C. Vollhardt, "Organic Chemistry," California University Press, Berkeley, CA, 1987.

23. P. D. Hong and J. H. Chen, Polymer, 40, 4077 (1999).

24. P. L. Flory, J. Chem. Phys., 15, 397 (1947).

25. J. Francois, J. Y. S. Gan, and J. M. Guenet, Macromolecules, 19, 2755 (1986).

26. H. Tanaka and T. Nishi, J. Chem. Phys., 82, 4326 (1985).

27. J. G. Powles and J. H. Strange, Proc. Phys. Soc., 82, 6 (1963).

28. P. D. Hong and J. H. Chen, Polymer, 39, 5809 (1998).

29. D. B. Frank, P. Stephen, and R. F. Kenneth, J. Colloid Interface Sci., 113, 336 (1986).

30. K. Ogino and H. Sato, J. Appl. Polym. Sci., 58, 1015 (1995).

31. A. Abragam, "The Principles of Nuclear Magnetism," Oxford University Press, London, 1961.

32. P. G. de Gennes, "Scaling Concepts in Polymer Physics," Cornell University Press, Ithaca, N. Y., 1979.

33. I. H. Park and C. S. Johnson, Macromolecules, 23, 1548 (1990).

34. S. J. Gibbs and C. S. Johnson, Macromolecules, 24, 6110 (1991).

35. P. Kubisa and O. Vogl, J. Polym. Sci., Polym. Lett. Ed., 13, 603 (1975).

36. N. A. Rotstein and T. P. Logde, Macromolecules, 25, 1316 (1992).

37. F. D. Blum, S. Pickup, and K. R. Foster, J. Colloid Interface Sci., 113, 336 (1986).

38. L. M. Wheeler and T. P. Lodge, Macromolecules, 22, 3399 (1989).

39. S. Matsukawa and I. Ando, Macromolecules, 29, 7136 (1996).

40. M. Nyden, O. Soderman, and G. Karlstrom, Macromolecules, 32, 127 (1999). 